\title{
Immunohistochemical detection of FGF-23 protein in tumors that cause oncogenic osteomalacia
}

\author{
Tobias Larsson ${ }^{1,3}$, Richard Zahradnik ${ }^{2}$, Jeffrey Lavigne ${ }^{2}$, Östen Ljunggren ${ }^{1}$, \\ Harald Jüppner ${ }^{3}$ and Kenneth B Jonsson ${ }^{1,3}$ \\ ${ }^{1}$ Department of Medical Sciences, University Hospital, Uppsala, Sweden, ${ }^{2}$ Immutopics Inc., 929 Calle Negocio, Suite A, San Clemente, \\ California 92673, USA and ${ }^{3}$ Endocrine Unit, Massachusetts General Hospital and Harvard Medical School, Boston, Massachusetts O2114, USA \\ (Correspondence should be addressed to K B Jonsson, Department of Medical Sciences, University Hospital, SE-751 85 Uppsala, Sweden; \\ Email: Kenneth.Jonsson@medsci.uu.se)
}

\begin{abstract}
Objective: Oncogenic hypophosphatemic osteomalacia (OOM) is a rare disease characterized by hypophosphatemia, inappropriately low levels of circulating 1,25-dihydroxyvitamin $\mathrm{D}_{3}$ and osteomalacia. The disease is most commonly caused by benign mesenchymal tumors that produce, among several other factors, fibroblast growth factor-23 (FGF-23). Current evidence thus suggests that this protein has an important role in the regulation of phosphate homeostasis. By producing polyclonal antibodies against human FGF-23 protein we wanted to determine the localization of FGF-23 protein in OOM tumors that express FGF-23 mRNA.

Design and methods: Three polyclonal antibodies were raised in rabbits against three different peptides with sequences derived from human FGF-23: [Cys-70]FGF-23(51-69)amide, [Tyr-223]FGF23(206-222)amide and [Tyr-224]FGF-23(225-244)amide. One of the resulting antisera was subsequently used for immunohistochemistry on sections from five different tumors causing OOM. FGF-23 mRNA expression was confirmed with in situ hybridization.

Results: After affinity purification, two of three antisera detected recombinant human FGF-23 by Western blot analysis. Through immunohistochemical analysis using the anti-[Tyr-224]FGF23(225-244)amide antibody and through in situ hybridization using full-length antisense FGF-23 cRNA as a probe, we showed that abundant amounts of FGF-23 protein and mRNA are present in certain tumor cells of five different OOM tumors.

Conclusions: We conclude that OOM tumors express FGF-23 protein and that the immunohistochemical detection of FGF-23 in OOM tumors is feasible and may help in establishing the diagnosis of tumor-induced hypophosphatemia through analysis of biopsies or surgical specimens.
\end{abstract}

European Journal of Endocrinology 148 269-276

\section{Introduction}

Oncogenic hypophosphatemic osteomalacia (OOM) is a rare acquired disorder caused by benign mesenchymal tumors that can be found in a wide range of locations, although the lower extremities and the craniofacial regions appear to be more frequently affected (1). The clinical and radiological findings in OOM include muscle pain and weakness, spontaneous fractures, and/or osteomalacia, while the biochemical characteristics include hypophosphatemia due to decreased renal reabsorption of phosphate, and inappropriately low levels of circulating 1,25-dihydroxyvitamin D3 $(1,2)$. These laboratory findings and clinical symptoms are similar to those observed in patients affected by X-linked hypophosphatemic rickets (XLH), a genetic disorder caused by inactivating mutations in PHEX (for phosphate-regulating gene with homology to endopeptidases located on the X-chromosome (3)), and autosomal dominant hypophosphatemic rickets (ADHR) (4).

Efforts to identify the genetic cause of ADHR through positional cloning recently led to the discovery of fibroblast growth factor-23 (FGF-23) (4), a 251 amino acid protein with a hydrophobic leader sequence, which is therefore readily secreted when expressed in several different clonal cell lines $(4,5)$. Independently, the cDNA encoding FGF-23 was also isolated through the analysis of a large number of cDNAs derived from OOM tumors (5) and through a homology-based search of genomic databases combined with amplification of cDNA by PCR using a mouse skin cDNA library (6).

OOM tumors were recently shown to express abundant amounts of the mRNA encoding FGF-23 (4, 5, 7 ), but the messages encoding several other proteins, including matrix extracellular protein and PHEX, 
were also readily detectable $(8,9)$. When administered i.p. in mice, recombinant FGF-23 increased urinary phosphate excretion, which resulted in hypophosphatemia and the development of rickets (5). Similar laboratory changes were observed in nude mice implanted s.c. with Chinese hamster ovary $(\mathrm{CHO})$ cells that stably expressed FGF-23 (5) and in transgenic mice expressing FGF-23 under the control of the systemic CAG promoter (cytomegalovirus enhancer coupled to a chicken $\beta$-actin promoter) (10). In these latter animals, expression of the type IIa sodium-dependent phosphate transport protein $\mathrm{Na}$ /P.IIa was dramatically reduced (10). Since Npt-2 is thought to be responsible for the bulk of phosphate reabsorption in the proximal tubules, reduced expression of this transporter provided a plausible explanation for the observed biochemical and histological findings in these mice, and is thus consistent with the hypothesis that FGF-23 is involved in the regulation of renal phosphate handling.

It remains uncertain, however, whether FGF-23 acts directly at the proximal tubular cells or whether its effect is indirect. In fact, in vitro experiments to assess the effects of FGF-23 on proximal tubular opossum kidney cells have thus far provided inconsistent results, since an inhibitory effect of recombinant FGF-23 on phosphate transport was observed by some investigators, while others, using slightly different experimental conditions, failed to document an effect $(5,11)$. Recently, FGF-23 was reported to, in combination with heparin, bind to FGF receptor 3, thereby initiating inhibition of renal phosphate reabsorption by the activation of a mitogen-activated protein kinase cascade, suggesting a direct renal effect (12).

Since FGF-23 seems to play a key role in the pathogenesis of OOM, the detection of FGF-23 protein in biopsies or surgical specimens could provide a significant advantage in the management of individuals affected by this disease. For the present study, we therefore developed antibodies towards human FGF-23 (hFGF-23), which after affinity purification proved useful for the immunohistochemical detection of FGF-23 in OOM tumor cells. These findings were confirmed through in situ hybridizations that revealed abundant expression of FGF-23 mRNA in the tumor cells, but not in surrounding tissue. We furthermore showed that affinity-purified antibodies recognizing different epitopes of $\mathrm{hFGF}-23$ readily detected the recombinant protein in conditioned medium from COS-7 cells transiently transfected with plasmid DNA encoding FGF-23.

\section{Materials and methods}

\section{Tumor material}

The five tumors used in this study were from different OOM patients who have been previously described (4). Consistent with the diagnosis of OOM, all patients presented with clinical and biochemical findings of hypophosphatemia due to urinary phosphate wasting, which resolved upon surgical tumor resection. Tumor 1 was from the left thigh (hemangiopericytoma) of a 54-year-old Japanese male (13). Tumor 2 was from the mandible (mixed connective tissue tumor) of a 31-year-old Korean female (14). Tumor 3 was from the left thigh (angiodysplasia) of a 31-year-old Swedish male (15). Tumor 4 was from the sole of the foot (hemangiopericytoma) of a German female (4). Tumor 5 was from the nose (hemangiopericytoma) of a 49-year-old Swedish female (4). All tumors had been frozen in liquid nitrogen and kept at $-70{ }^{\circ} \mathrm{C}$ until analysis. Small pieces of the tumor were removed and embedded in paraffin before sectioning with a microtome for in situ hybridization and immunohistochemistry.

\section{Production of FGF-23-specific antibodies}

Synthetic peptides corresponding to sequences from different portions of hFGF-23 protein were synthesized at the Biopolymer Core Facility of the Massachusetts General Hospital using an Applied Biosystems synthesizers (model 430A or 431A; Applied Biosystems, Foster City, CA, USA) and F-moc chemistry. These peptides, [Cys-70]FGF-23(51-69)amide, [Tyr-223] FGF-23(206-222)amide and [Tyr-224]FGF-23(225244)amide, were purified to homogeneity by reversephase HPLC, and their sequences were confirmed by amino acid composition analysis, amino acid sequence analysis, and mass spectroscopy. A portion of each peptide was conjugated to keyhole limpet hemocyanin and emulsified in Freund's adjuvant before intracutaneous injection into rabbits at Cocalico Biologicals (Reamstown, PA, USA). Each rabbit received booster injections after 14, 21 and 49 days, and was bled every 2-4 weeks. For affinity purification, each of the three different FGF-23 synthetic peptides was covalently coupled to a gel support $(3 \mathrm{mg} / 2 \mathrm{ml}$ gel; $4 \%$ cross-linked agarose, AminoLink Kit; Pierce Chemical, Rockford, IL, USA). Anti-FGF-23 immunoglobulins were purified by passing approximately $30 \mathrm{ml}$ (one bleed) of off-the-clot antiserum over the appropriate immobilized peptide column equilibrated with $0.01 \mathrm{~mol} / \mathrm{l} \mathrm{PBS}, \mathrm{pH} 7.4$. The column was rinsed with the same buffer to remove unbound material and then eluted with $0.2 \mathrm{~mol} / \mathrm{l}$ glycine-HCl, $\mathrm{pH}$ 2.3. The recovered antibody was neutralized with $1 \mathrm{~mol} / \mathrm{l}$ Tris and adjusted to a normal saline concentration. The affinity-purified antibodies were then diluted with $0.01 \mathrm{~mol} / \mathrm{l}$ PBS to a protein concentration of $25 \mu \mathrm{g} / \mathrm{ml}$ and stored at $2-8^{\circ} \mathrm{C}$.

\section{Expression of recombinant FGF-23 protein}

The cDNA encoding wild-type hFGF-23 was isolated by PCR from a cDNA library derived from tumor 1 (forward primer: TCA GAG CAG GGC ACG ATG TTG; 
reverse primer: GAT GAA CTT GGC GAA GGG GC). The PCR product was ligated into the vector pCDNA3.1-V5His-TOPO (Invitrogen Corporation, Carlsbad, CA, USA) to yield full-length hFGF-23 tagged with V5/His at the carboxyl-terminus, [V5-His]rhFGF-23(1-251). The R176Q mutation (4) was introduced by the QuickChange site-directed mutagenesis procedure (Stratagene, La Jolla, CA, USA) as described in the supplier's manual to yield [V5-His;R176Q]rhFGF-23(1-251). Anticipated nucleotide sequence and insert orientation were confirmed by nucleotide sequence analysis. Both plasmids were transfected into COS-7 cells using Effectene transfection reagent (Qiagen, Valencia, CA, USA). Conditioned medium from transfected cells was collected $24-72 \mathrm{~h}$ after transfection and stored at $-20^{\circ} \mathrm{C}$ until experiments were performed.

\section{Western blot analysis}

Conditioned medium from transfected COS-7 cells and protein standards with known molecular masses were electrophoresed through $12 \%$ SDS-PAGE minigels (Bio-Rad, Hercules, CA. USA) and electroblotted onto Immobilon-P membranes (Millipore, Bedford, MA, USA). Membranes were blocked with $5 \%$ dried milk in PBS, pH 7.4, containing 0.1\% Tween before incubation with either crude anti-FGF-23 antisera (diluted 1:100 in $5 \%$ milk in PBS) or $1 \mu \mathrm{g} / \mathrm{ml}$ affinity-purified antihFGF-23 antibodies diluted in PBS with 5\% dried milk and $0.1 \%$ Tween. After $12 \mathrm{~h}$ of incubation at $4{ }^{\circ} \mathrm{C}$, the membrane was rinsed with PBS and incubated with an horseradish peroxidase (HRP)-coupled goat antirabbit antibody (Dako, Dakopatt AB, Älvsjö, Sweden) (diluted 1:1000 in 5\% milk in PBS). After $2 \mathrm{~h}$ at room temperature, the membrane was rinsed three times with PBS before visualizing immunoreactive hFGF-23 by enhanced chemiluminescence (Amersham, Piscataway, NJ, USA). In some experiments, an HRPconjugated anti-V5 antibody (Invitrogen) was used at a dilution of 1:1000, to serve as a positive control for the detection of [V5-His]rhFGF-23(1-251) and [V5His;R176Q]rhFGF-23(1-251).

\section{In situ hybridization}

${ }^{35}$ S-labeled sense and antisense cRNA probes for
hFGF-23 were transcribed from a linearized plasmid
encoding full-length hFGF-23 using either SP6 or T7
RNA polymerase. The probes were purified by gel fil-
tration through a Micro Bio-Spin chromatography
column (Bio-Rad). Hybridization of deparaffinized sec-
tions from tumors 1 to 5 was performed as previously
described (16). The slides were then covered with
photographic emulsion and exposed for 7 days at
$4^{\circ} \mathrm{C}$. Slides were developed and stained with hema-
toxylin/eosin (H\&E).

\section{Immunohistochemistry}

After deparaffination tissue sections were blocked with $0.3 \%$ hydrogen peroxidase in methanol for $15 \mathrm{~min}$. Further blocking was performed with normal goat serum (Dako) diluted 1:10 in PBS for $15 \mathrm{~min}$, avidin for $15 \mathrm{~min}$ and biotin for $15 \mathrm{~min}$ (Avidin-Biotin Blocking Kit; Vector Laboratories, Burlingame, CA, USA). Between each blocking step the slides were rinsed three times in PBS. The slides were then incubated for $1.5 \mathrm{~h}$ with primary rabbit anti-hFGF-23 antibody to [Tyr-224]FGF-23(225-244)amide (50 $\mu \mathrm{l} / \mathrm{section}$, diluted to $0.25 \mu \mathrm{g} / \mathrm{ml}$ in PBS), before rinsing three times with PBS and incubation for $30 \mathrm{~min}$ with secondary biotinylated goat anti-rabbit antibody (Amersham) diluted 1:200 in PBS. For visualization, Vectastain ABC Kit (Vector Laboratories) was used according to the manufacturer's instructions. Slides were counterstained with Mayer's hematoxylin.

\section{Results}

\section{Characterization of polyclonal antibodies}

To generate antisera directed against hFGF-23, rabbits were immunized with three different synthetic fragments of this recently discovered protein. Only the rabbits immunized with [Tyr-223]FGF-23(206-222)amide and [Tyr-224]FGF-23(225-244)amide yielded crude

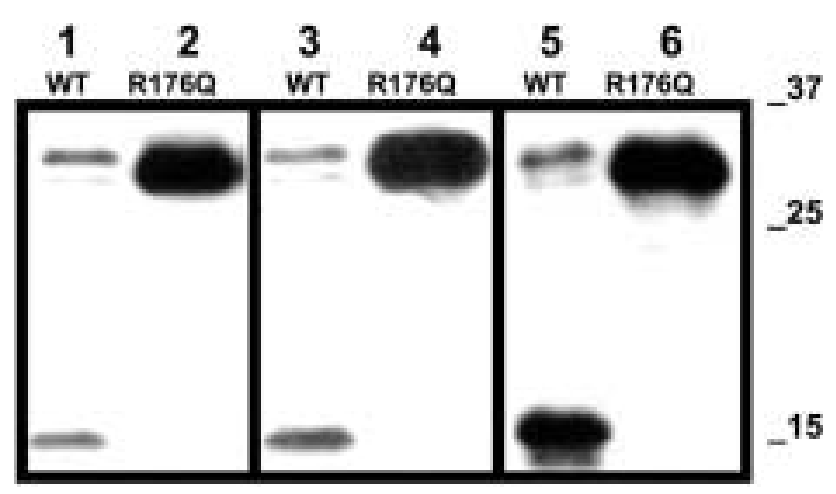

Figure 1 Western blot analysis of recombinant FGF-23. COS-7 cells were transfected with the plasmid encoding either [V5His]rhFGF-23(1-251) or [V5-His;R176Q]rhFGF-23(1-251). Conditioned media were collected $48 \mathrm{~h}$ after transfection and $10 \mu \mathrm{l}$ were analyzed by Western blotting. The three different affinitypurified antibodies were used as follows: the HRP-conjugated anti-V5 antibody was used at an dilution of $1 / 10000$ and exposure time was approximately $3 \mathrm{~min}$ (lanes 1 and 2 ). The antisera raised against [Tyr-223]FGF-23(206-222)amide and

[Tyr-224]FGF-23(225-244)amide were used at a dilution of $1 / 10000$ and exposure time was approximately 1 min (lanes 3 and 4; and lanes 5 and 6 respectively). All antibodies detected two bands of $\sim 32 \mathrm{kDa}$ and $\sim 16 \mathrm{kDa}$ in media conditioned by COS-7 cells expressing the wild-type (WT) plasmid. In media conditioned by cells expressing the plasmid containing the R176Q mutation only the $32 \mathrm{kDa}$ band was detected. 
A

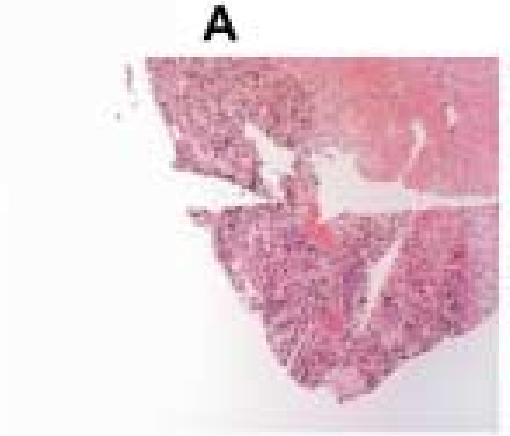

2:1
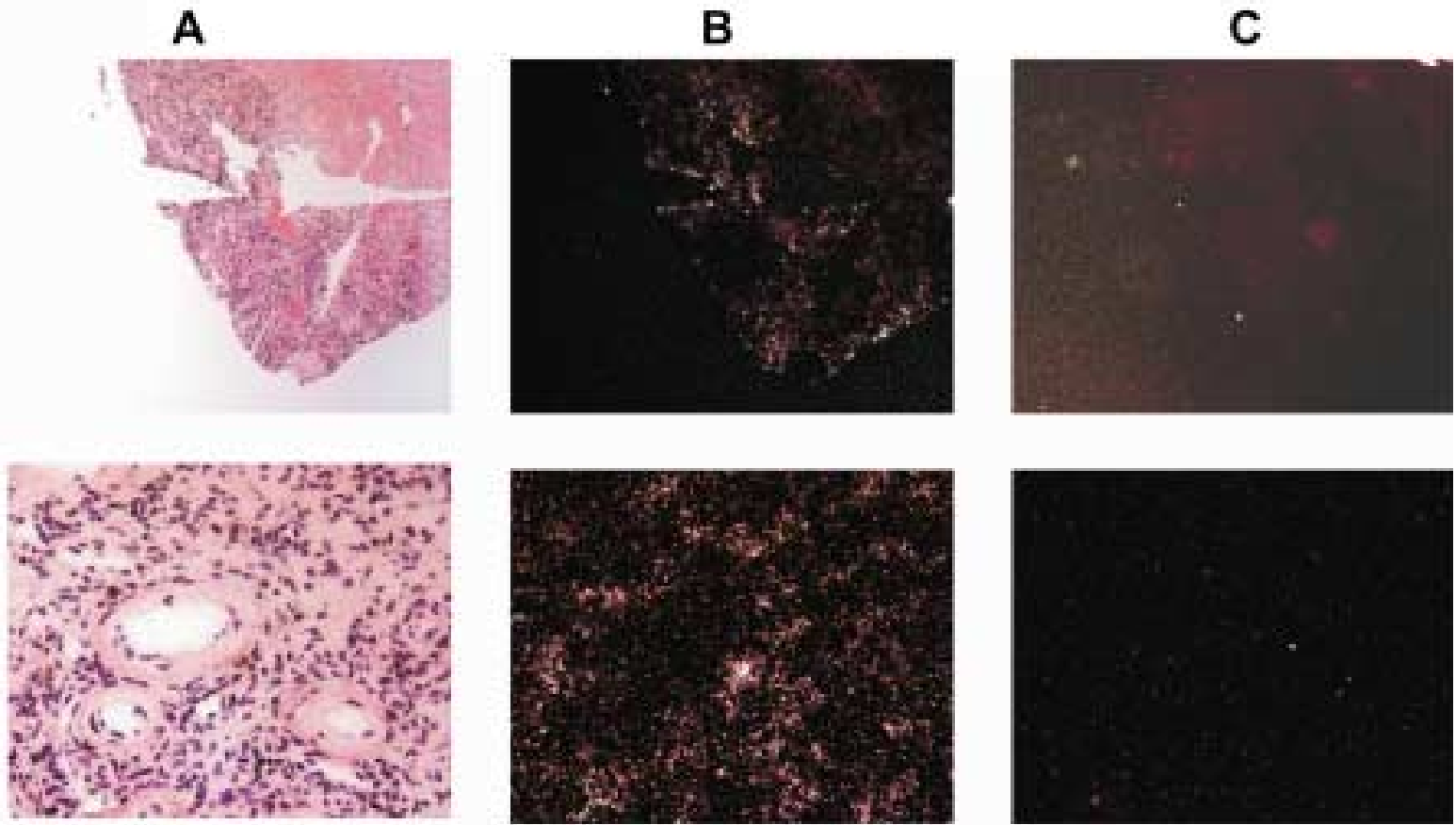

2:2

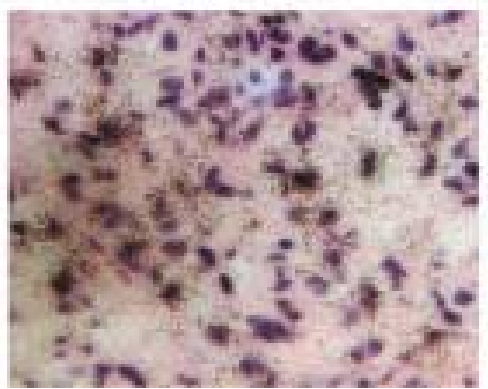

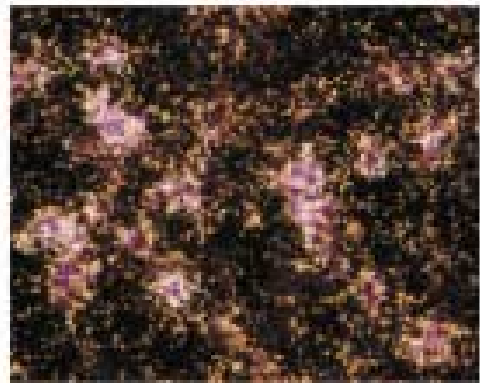

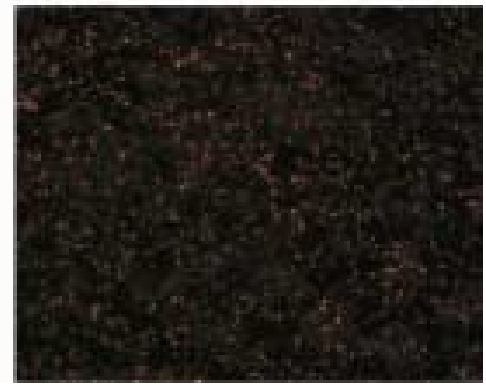

Figure 2 In situ hybridization of OOM tumors. Deparaffinized sections of tumors that caused OOM were hybridized with FGF-23 cRNA sense or antisense probe as described in Materials and methods. The sections were counterstained with H\&E. Panel 2.1 shows tumor 4 (hemangiopericytoma): (A) in situ hybridization using the FGF-23 antisense probe-bright field; (B) in situ hybridization using FGF-23 antisense probe-dark field; (C) in situ hybridization using the FGF-23 sense probe; $\times 10$ magnification. Panel 2.2 shows tumor 3 (angiodysplasia); $\times 50$ magnification. Panel 2.3 shows tumor 5 (hemangiopericytoma); $\times 100$ magnification.

antisera with sufficiently high affinity to allow detection by Western blot analysis of [V5-His]rhFGF-23(1-251) in conditioned medium from transfected $\operatorname{COS}-7$ cells. Thus, at a dilution of $1: 100$, both the latter antisera detected the same 32 and $16 \mathrm{kDa}$ protein bands that were also observed when using the anti-V5 antibody (data not shown).

To improve sensitivity of these Western blots, antibodies against the FGF-23 fragments were affinity purified using the respective synthetic peptides coupled to Sepharose 4B. While conditioned medium from mocktransfected COS-7 cells revealed no immunoreactive protein with either of the three antibodies, immunoreactive material was detected in medium from COS-7 cells transfected with [V5-His]rhFGF-23(1-251) (Fig. 1). The antibody directed against [Cys-70]FGF23(51-69)amide proved to have a very low affinity for recombinant FGF-23. When used in high concentrations and with prolonged exposure times, recombinant material was detected but the finding was inconsistent and the antibody did not provide useful information on the amino-terminal fragments of FGF-23 (data not shown). The antibodies directed against [Tyr-224]FGF-23(225-244)amide and [Tyr223]FGF-23(206-222)amide detected two protein bands in conditioned media from cells expressing [V5His]rh FGF-23(1-251); a $\sim 16 \mathrm{kDa}$ band and a $\sim 32 \mathrm{kDa}$ protein band, presumably representing the carboxyl-terminal fragment of FGF-23 and the intact protein respectively (Fig. 1) This agrees with recent data showing that full-length FGF-23 is cleaved at position 179 when expressed in CHO cells (17).

When COS-7 cells were transfected with the plasmid encoding FGF-23 carrying the R176Q mutation identified in patients with ADHR (4), both anti-FGF-23 antibodies detected only the full-length protein, which was 
A
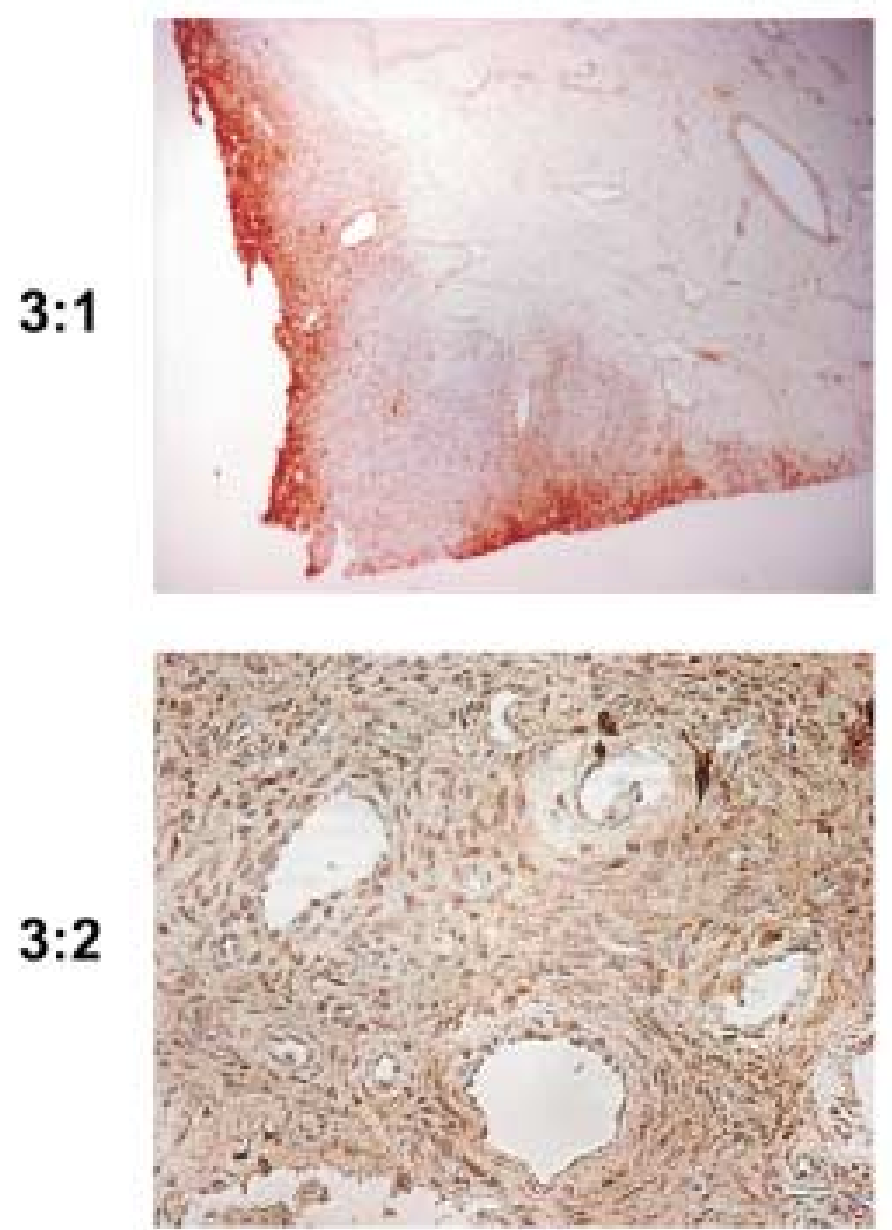

$3: 2$
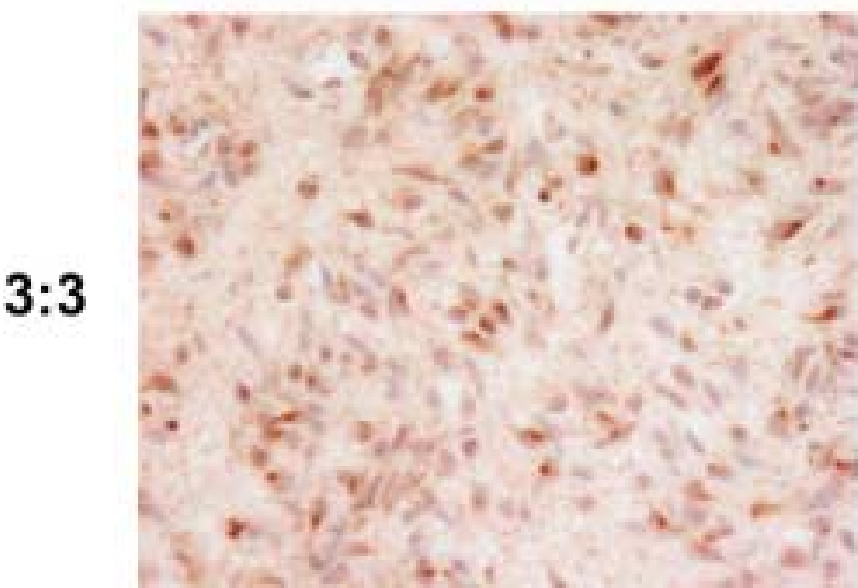

B
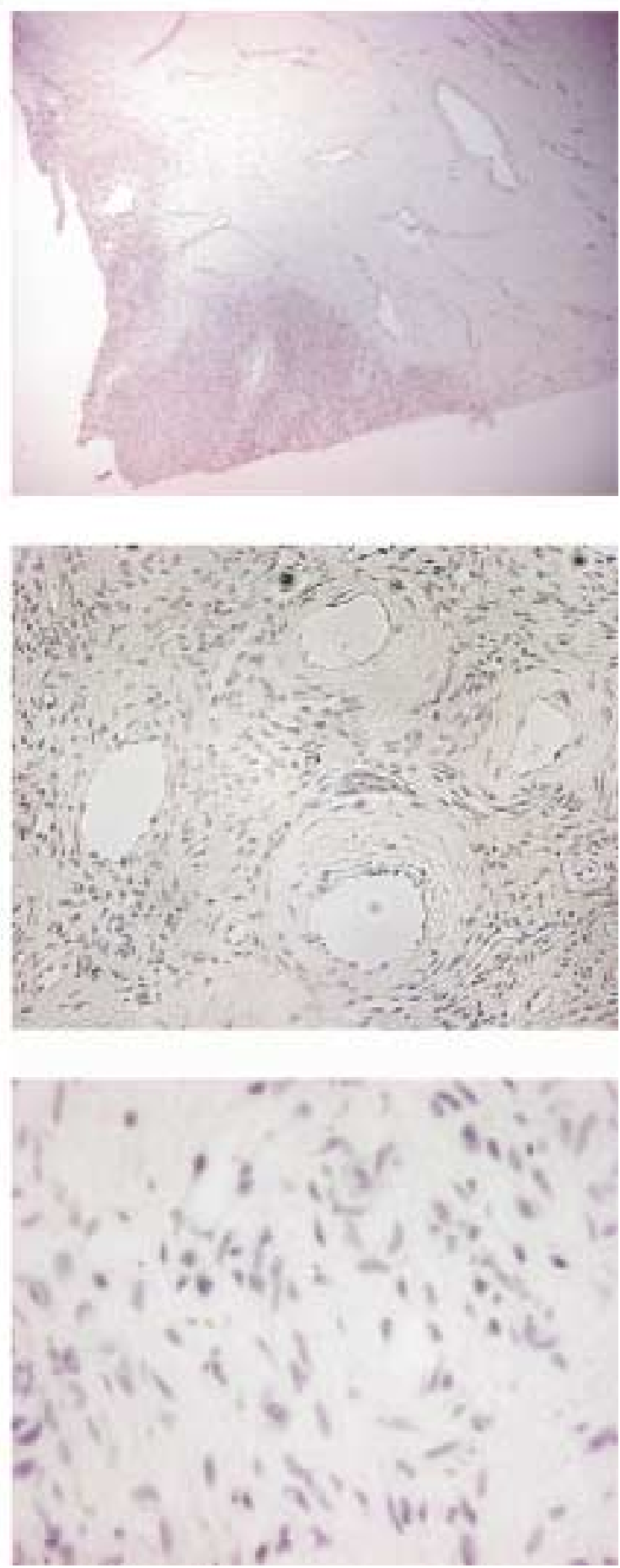

Figure 3 Immunohistochemistry of OOM tumors. Deparaffinized sections of tumors that caused OOM were analyzed by immunohistochemistry with the anti-[Tyr-224]FGF-23(225-244) antibodies as described in Materials and methods. The sections were counterstained with Mayer's hematoxylin. Panel 3.1 shows tumor 4 (hemangiopericytoma): (A) positive immunohistochemical signal; (B) adjacent section incubated with excess of peptide before staining; $\times 10$ magnification. Panel 3.2 shows tumor 3 (angiodysplasia); $\times 40$ magnification. Panel 3.3 shows tumor 5 (hemangiopericytoma); $\times 80$ magnification. 
also detected by the antibody against the V5 epitope (Fig. 1). Taken together these data indicated that both antisera recognize the full-length protein as well as the carboxyl-terminal portion of the molecule. Furthermore, the anti-FGF-23 antibodies readily recognized a mutant FGF-23 containing one of the ADHR mutations (R176Q), and consistent with findings by others the introduction of this mutation prevented cleavage into an amino- and an carboxyl-terminal fragment (18).

\section{OOM tumors express FGF-23 mRNA and the encoded protein}

To determine whether OOM tumors express the mRNA encoding FGF-23, in situ hybridizations were performed on sections of five different tumors using a radiolabeled FGF-23 cRNA antisense probe and, as negative control, a sense probe. In all five tumors, specific hybridization was observed, which was distributed in clusters that were localized within the tumor cell population (Fig. 2). In greater magnification, hybridization was observed in and in the proximity of some, but not all, cells within this cell population. No labeling was found in any of the five tumors using the sense probe (Fig. 2).

Paraffin sections from the same tumors that were used for in situ hybridizations were used to assess FGF-23 protein expression by immunohistochemistry. Using the affinity-purified anti-[Tyr-224]FGF-23(225244)amide antibody, all five tumors showed a readily detectable signal with a distribution similar to that observed by in situ hybridizations using the FGF-23 antisense probe (Fig. 3). Although our five tumors were given somewhat different pathological diagnoses, they all had a similar structure within the tumor cell areas. The tumors had been narrowly excised and it was not possible to see much of the supporting tissues, i.e. skin, bone or muscle. However, the samples contained some capsular connective tissue structures that enclosed clusters of small cells that did not seem to invade the surrounding connective tissue. The cells were embedded in a rich stroma; they exhibited some features typical of tumoral growth, such as difference in cellular size, nuclear atypia and a somewhat disorganized growth pattern. Some, but not all, of the tumor cells were strongly stained and FGF-23 showed mainly an intracellular localization even though some staining was seen also in direct proximity to the stained tumor cells (Fig. 3). We conclude from this that it is the tumor cells that expressed both the FGF-23 mRNA and the protein, but that only a portion of the total number of tumor cells are responsible for this production.

Preincubation of the antibodies with excess amounts of peptide used for immunization completely blocked the signal, thus providing evidence for the specificity of the antibody (Fig. 3). The affinity-purified antibodies against [Tyr-223]FGF-23(206-222)amide failed to generate, under the described conditions, a specific signal (data not shown).

\section{Discussion}

In ADHR, mutations in FGF-23 lead to urinary loss of phosphate and consequently hypophosphatemia and osteomalacia (4). ADHR shares significant clinical and laboratory similarities with OOM. Using the five OOM tumors used in this reports we previously demonstrated abundant expression of FGF-23 mRNA by Northern blot analysis (4) suggesting that FGF-23 is the phosphaturic factor responsible for the clinical findings in both groups of patients. This hypothesis is supported by in vivo findings in mice that received recombinant FGF-23 i.p. (5) and by findings in transgenic animals overexpressing FGF-23 under the control of the CAG promoter (10). Hypophosphatemia due to renal phosphate wasting also occurs in patients with XLH, a disorder in which the membrane-bound endopeptidase PHEX is inactivated by one of numerous different mutations introduced into this X-chromosomal gene (19). In vitro translated full-length PHEX was recently shown to cleave wild-type recombinant FGF-23, but not FGF-23 carrying one of the three known ADHR mutations (11). Although these findings were not confirmed in another study using a full-length PHEX product encoded by a recombinant baculoviral vector (20) FGF-23 is likely to contribute, directly or indirectly, to renal phosphate handling.

In this report, we now describe the production and characterization of three different polyclonal antisera raised against different portions of FGF-23. Specificity antibodies were demonstrated by Western blot analysis of [V5-His]rhFGF-23(1-251) which was cleaved into an amino-terminal FGF-23 fragment and a carboxylterminal fragment detected by the two antibodies raised toward [Tyr-224]FGF-23(225-244)amide or [Tyr-223]FGF-23(206-222)amide. However, in agreement with data from others, the protein bands comprising the carboxyl-terminal FGF-23 fragment disappeared upon introduction of the ADHR mutation.

The observation that the three known mutations in FGF-23 that cause ADHR are located within a consensus site for cleavage by the subtilisin-like proprotein convertases (RXXR), suggested that the pathogenesis of ADHR could involve altered processing of the protein. Accordingly, it was recently reported that, when using a carboxyl-terminal antibody as the means of detection, hFGF-23 migrated as $\sim 32 \mathrm{kDa}$ and $\sim 12 \mathrm{kDa}$ species when produced in HEK cells. However, when the $\mathrm{R} 176 \mathrm{Q}$ or R179W mutation was present only the full-length protein was detected (18). Furthermore, it was recently demonstrated that only full-length FGF-23 causes hypophosphatemia in vivo (17). These results indicate that the cleavage between Arg179 and Ser180 is the primary processing of FGF-23 that 
converts biologically active FGF-23 protein into an inactive form. The ADHR mutations may therefore lead to increased circulating concentrations of FGF-23 in patients afflicted with this disease. In support of this hypothesis we consistently observed higher expression levels of the full-length protein in cultures of COS-7 cells transfected with [V5-His;R176Q]rhFGF23(1-251) than with wild-type FGF-23.

We showed that FGF-23 can be detected immunohistochemically using an affinity-purified antibody toward [Tyr-224]FGF-23(225-244)amide in tumors that cause OOM. The previously described antiserum raised against residues 206-222 of hFGF-23 (4) is similar to the anti-[Tyr-223]FGF-23(206-222) antiserum used in the present report, and both antisera detected recombinant FGF-23 produced in COS-7 cells. However, the [Tyr-223]FGF-23(206-222) antiserum was unable to produce a clear specific signal when used for immunohistochemical analysis of OOM tumors. These negative findings are most probably due to either the relatively low affinity of the antibody for FGF-23 or the unavailability of this antigen during the experimental conditions used in our study. Using the antibody raised against [Tyr-224]FGF-23(225244)amide, we were able to show specific FGF-23 staining in all five available OOM tumors, and furthermore expression of FGF-23 mRNA was confirmed in sections from the same tumor block by in situ hybridization. Both methods thus revealed that FGF-23 expression is found in clustered tumor cells, making it likely that the availability of the described anti-FGF-23 antibodies, or other antibodies with similar specificity, will be useful in establishing the diagnosis of OOM. Our findings may be particularly important for OOM caused by polytumoral disease where immunohistochemical analysis of removed tissue would help in deciding the subsequent treatment strategy (21). Furthermore, we have recently shown that the anti-[Tyr-223]FGF23(206-222) and anti-[Tyr-224]FGF-23(225-244) antisera can be combined in a sensitive ELISA for the detection of FGF-23 in the circulation of humans. With this assay we detected increased circulating FGF-23 levels in patients with XLH and OOM (22).

It has been reported earlier that FGF-23 mRNA transcripts are expressed at very low levels in a limited number of human and mouse tissues, and RT-PCR is typically required to detect the transcript in tissues such as heart, liver and thyroid gland (4). Using the antibodies toward [Tyr-224]FGF-23(225-244)amide, we examined a number of control tissues and did not detect a specific signal in parathyroid adenoma, liver or a gastrointestinal carcinoid tumor. The natural source of FGF-23 therefore remains uncertain.

In conclusion, using immunohistochemical analysis and in situ hybridizations, we have confirmed FGF-23 mRNA and protein expression in tumors giving rise to OOM. However, the described antisera may not only be useful for analysis of surgical specimens but have also proved useful in the development of an immunoassay for detection of FGF-23 in the circulation.

\section{Acknowledgements}

We would like to thank Melissa Knight, Janet Saxton, Anna-Lena Johansson, Birgitta Bondesson and Andreas Larsson for skillful technical assistance; Ashok Khatri for peptide synthesis; and Ernestina Schipani, for fruitful discussions and suggestions. The study was supported by The Swedish Foundation for International Cooperation in Research and Higher Education and the Tore Nilsson Foundation.

\section{References}

1 Ryan EA \& Reiss E. Oncogenous osteomalacia. Review of the world literature of 42 cases and report of two new cases. American Journal of Medicine 198477 501-512.

2 Nelson AE, Mason RS \& Robinson BG. The PEX gene: not a simple answer for X-linked hypophosphataemic rickets and oncogenic osteomalacia. Molecular and Cellular Endocrinology 1997132 $1-5$.

3 A gene (PEX) with homologies to endopeptidases is mutated in patients with X-linked hypophosphatemic rickets. The HYP Consortium. Nature Genetics 199511 130-136.

4 White KE, Jonsson KB, Carn G, Hampson G, Spector TD, Mannstadt $\mathrm{M}$ et al. The autosomal dominant hypophosphatemic rickets (ADHR) gene is a secreted polypeptide overexpressed by tumors that cause phosphate wasting. Journal of Clinical Endocrinology and Metabolism $2001 \mathbf{8 6}$ 497-500.

5 Shimada T, Mizutani S, Muto T, Yoneya T, Hino R, Takeda S et al. Cloning and characterization of FGF23 as a causative factor of tumor-induced osteomalacia. PNAS 200198 6500-6505.

6 Yamashita T, Yoshioka M \& Itoh N. Identification of a novel fibroblast growth factor, FGF-23, preferentially expressed in the ventrolateral thalamic nucleus of the brain. Biochemical and Biophysical Research Communications 2000277 494-498.

7 Seufert J, Ebert K, Muller J, Eulert J, Hendrich C, Werner E et al. Octreotide therapy for tumor-induced osteomalacia. New England Journal of Medicine 2001345 1883-1888.

8 Jan de Beur SM, Barberio D, Cook B, Estes S, Finnegan R, Madden $\mathrm{S}$ et al. Gene expression profiles of tumors associated with oncogenic osteomalacia. Journal of Bone Mineral Research 200015 S357.

9 Rowe PS, de Zoysa PA, Dong R, Wang HR, White KE, Econs MJ et al. MEPE, a new gene expressed in bone marrow and tumors causing osteomalacia. Genomics 200067 54-68.

10 Shimada T, Yoneya T, Hino R, Takeuchi Y, Fukumoto S \& Yamashita T. Transgenic mice expressing fibroblast growth factor 23 (FGF23) demonstrate hypophosphatemia with low serum 1,25dihydroxyvitamin D $[1,25(\mathrm{OH}) 2 \mathrm{D}]$ and rickets/osteomalacia. Journal of Bone Mineral Research 200116 S151.

11 Bowe AE, Finnegan R, Jan de Beur SM, Cho J, Levine MA, Kumar $\mathrm{R}$ et al. FGF-23 inhibits renal tubular phosphate transport and is a PHEX substrate. Biochemical and Biophysical Research Communications $2001 \mathbf{2 8 4} 977-981$.

12 Yamashita T, Konishi M, Miyake A, Inui K \& Itoh N. Fibroblast growth factor (FGF)-23 inhibits renal phosphate reabsorption by activation of the mitogen-activated protein kinase pathway. Journal of Biological Chemistry 2002277 28265-28270.

13 Miyauchi A, Fukase M, Tsutsumi M \& Fujita T. Hemangiopericytoma-induced osteomalacia: tumor transplantation in nude mice causes hypophosphatemia and tumor extracts inhibit renal 25hydroxyvitamin D 1-hydroxylase activity. Journal of Clinical Endocrinology and Metabolism 198867 46-53. 
14 Yang IM, Park YK, Hyun YJ, Kim DY, Woo JT, Kim SW et al. Oncogenic osteomalacia caused by a phosphaturic mesenchymal tumor of the oral cavity: a case report. Korean Journal of Internal Medicine 199712 89-95.

15 Nelson AE, Mason RS, Robinson BG, Hogan JJ, Martin EA, Ahlstrom $\mathrm{H}$ et al. Diagnosis of a patient with oncogenic osteomalacia using a phosphate uptake bioassay of serum and magnetic resonance imaging. European Journal of Endocrinology $2001 \mathbf{1 4 5}$ 469-476.

16 Soegiarto DW, Kiachopoulos S, Schipani E, Juppner H, Erben RG \& Lanske B. Partial rescue of PTH/PTHrP receptor knockout mice by targeted expression of the Jansen transgene. Endocrinology 2001 142 5303-5310.

17 Shimada T, Muto T, Urakawa I, Yoneya T, Yamazaki Y, Okawa K et al. Mutant FGF-23 responsible for autosomal dominant hypophosphatemic rickets is resistant to proteolytic cleavage and causes hypophosphatemia in vivo. Endocrinology $2002 \mathbf{1 4 3}$ 3179-3182.

18 White KE, Carn G, Lorenz-Depiereux B, Benet-Pages A, Strom TM \& Econs MJ. Autosomal-dominant hypophosphatemic rickets
(ADHR) mutations stabilize FGF-23. Kidney International 2001 $602079-2086$.

19 Sabbagh Y, Jones AO \& Tenenhouse HS. PHEXdb, a locus-specific database for mutations causing X-linked hypophosphatemia. Human Mutation 200016 1-6.

20 Guo R, Liu S, Spurney RF \& Quarles LD. Analysis of recombinant Phex: an endopeptidase in search of a substrate. American Journal of Physiology. Endocrinology and Metabolism 2001281 E837-E847.

21 Haviv YS \& Silver J. Late onset oncogenic osteomalacia-associated with neurofibromatosis type II. Clinical Nephrology $2000 \mathbf{5 4}$ 429-430.

22 Jonsson KB, Zahradnik R, Larsson T, White KE, Hampson G, Miyauchi A et al. FGF-23 Is a circulating factor that is elevated in oncogenic osteomalacia and X-linked hypophosphatemic rickets. Journal of Bone Mineral Research 200217 S158.

Received 5 June 2002

Accepted 23 October 2002 